\title{
T-817MA, but Not Haloperidol and Risperidone, Restores Parvalbumin-Positive $\gamma$-Aminobutyric Acid Neurons in the Prefrontal Cortex and Hippocampus of Rats Transiently Exposed to MK-801 at the Neonatal Period
}

\author{
Takashi Uehara, ${ }^{1,2}$ Tomiki Sumiyoshi, ${ }^{1}$ Tomonori Seo, ${ }^{1}$ Tadasu Matsuoka, ${ }^{1}$ \\ Hiroko Itoh, ${ }^{1}$ and Masayoshi Kurachi ${ }^{1}$ \\ ${ }^{1}$ Department of Neuropsychiatry, Graduate School of Medicine and Pharmaceutical Sciences, University of Toyama, \\ 2630 Sugitani, Toyama 930-0194, Japan \\ ${ }^{2}$ Division of Molecular and Clinical Neurobiology, Department of Psychiatry, Ludwig-Maximilians University of Munich, \\ Nußbaumstraße 7, 80336 Munich, Germany
}

Correspondence should be addressed to Takashi Uehara, uehara@med.u-toyama.ac.jp

Received 16 May 2012; Accepted 7 June 2012

Academic Editors: A. Deveci and B. A. Ola

Copyright ( $(2012$ Takashi Uehara et al. This is an open access article distributed under the Creative Commons Attribution License, which permits unrestricted use, distribution, and reproduction in any medium, provided the original work is properly cited.

\begin{abstract}
The number of parvalbumin (PV)-positive $\gamma$-aminobutyric acid (GABA) neurons is decreased in the brain of rats transiently exposed to MK-801, an N-methyl-D-aspartate (NMDA) receptor blocker, in the neonatal stage (Uehara et al. (2012)). T-817MA [1-\{3-[2-(1-benzothiophen-5-yl)ethoxy]propyl\} azetidin-3-ol maleate] is a neuroprotective agent synthesized for the treatment of psychiatric disorders characterized by cognitive disturbances, such as dementia. We herein sought to determine whether T817MA, haloperidol (HPD), or risperidone (RPD) would ameliorate the decrease in the number of PV-positive GABA neurons in the medial prefrontal cortex (mPFC) and hippocampus of the model animals. Rats were treated with MK-801 (0.2 mg/kg/day) or vehicle on postnatal days (PD) 7-10, and the number of PV-positive neurons in the MPFC and hippocampus were measured on PDs 63. T-817MA $(20 \mathrm{mg} / \mathrm{kg})$, HPD $(1 \mathrm{mg} / \mathrm{kg})$, or RPD $(1 \mathrm{mg} / \mathrm{kg})$ were administered during PDs $49-62$. Fourteen-day administration of T-817MA reversed the decrease in the number of PV-positive neurons in the above brain regions of rats given MK-801, whereas HPD and RPD were ineffective. These results indicate that T-817MA provides a novel pharmacologic strategy to enhance cognitive function in patients with schizophrenia.
\end{abstract}

\section{Introduction}

Schizophrenia is a complex and severe disorder, which affects approximately $1 \%$ of general population $[1,2]$. Patients with the illness manifest positive symptoms (e.g., delusions, hallucinations, thought disorder) and negative symptoms (e.g., anhedonia, blunted affect, social withdrawal), as well as disturbances in a range of cognitive domains, such as several types of memory, attention/information processing, executive functions, and verbal fluency [2]. The clinical course of schizophrenia is characterized by episodic positive symptoms and progressive negative and cognitive symptoms. The onset of illness is generally in the late adolescence or early adulthood, which is preceded by prodromal symptoms, including nonspecific mood symptoms, mild psychotic symptoms, cognitive impairment, and social withdrawal [3]. About one-third is resistant to treatment with existing antipsychotic drugs [4].

Schizophrenia is considered as a neurodevelopmental disorder $[5,6]$. Progressive pathophysiological processes possibly begin in the prodromal stage and continue after the onset of the illness $[3,7,8]$. Apoptosis (programmed cell death) may play a role in this process that leads to neurodegeneration. The vulnerability of neurons to proapoptotic insults (proapoptotic triggers) could produce selective dendritic and synaptic losses [9]. The pro-apoptotic 
stimuli related to the pathophysiology of schizophrenia include (1) glutamatergic excitotoxicity, (2) excessive synaptic calcium flux, (3) oxidative stress, and (4) reduced neurotrophin levels (e.g., brain-derived neurotrophic factor BDNF, neurotrophin-3 NT-3) [9]. Thus, increased calcium levels and oxidative stress can lead to glutamate excitotoxicity and promote apoptotic activity [10]. Genetic and environmental factors, such as viral infection, may affect neural circuits during adolescence or young adulthood, leading to the emergence of positive and negative symptoms [11, 12]. Progressive volume reduction of whole brain, especially left and right prefrontal cortex, is found in individuals at high genetic risk of schizophrenia who later develop schizophrenia [13]. Therefore, there is increasing interest in the potential role for neuroprotection in the action of antipsychotic drugs [14]. Some second-generation antipsychotic drugs (SGAs) may have advantages in this respect, whereas the first-generation antipsychotic drugs, such as haloperidol particularly in high doses, may be neurodegenerative [14].

Dysfunction of $\gamma$-aminobutyric acid (GABA) interneurons, particularly those containing the calcium-binding protein parvalbumin (PV), has been suggested to be associated with the pathophysiology of schizophrenia, through the consequent imbalance between excitation and inhibition in the cerebral cortex $[15,16]$. Abnormalities of GABA neurons are thought to be related to cognitive impairments of schizophrenia [15]. These considerations are consistent with the histological evidence for the reduction of PV-positive GABA interneuron density in the frontal cortex $[15,17,18]$ and hippocampus $[19,20]$ in subjects with schizophrenia.

T-817MA [1-\{3-[2-(1-benzothiophen-5-yl) ethoxy] propyl\} azetidin-3-ol maleate] is a novel neuroprotective agent (Figure 1). It (1) exerts neuroprotective effects against neurotoxicity caused by intracerebroventricular infusion of amyloid- $\beta(\mathrm{A} \beta)[21,22]$, (2) facilitates neurogenesis, such as neuron proliferation, neurite outgrowth, and synaptogenesis, through the increase of neurotrophic factors [23], and (3) improves cognitive impairment in rats receiving intracerebroventricular infusion of $\mathrm{A} \beta[21,22]$ or expressing FTDP17 human P301L mutant tau [24]. T-817MA has been shown to also ameliorate behavioral and histological abnormalities in rodent models of schizophrenia $[25,26]$.

We previously reported that 14-day treatment with T817MA ameliorated a decrease in the number of PV-positive GABA neurons in the medial prefrontal cortex (mPFC) and hippocampus of rats that received neonatal administration of MK-801, an N-methyl-D-aspartate (NMDA) receptor antagonist [25]. In this paper, we extend our studies by including haloperidol (HPD) and risperidone (RPD) as comparator compounds.

\section{Materials and Methods}

2.1. Animals. Preparation of the animal model was based on previous reports [25, 27-29]. Female Wistar rats obtained at 14 days of pregnancy (Japan SLC, Hamamatsu, Japan) were housed individually at $24 \pm 2^{\circ} \mathrm{C}$ under a light-dark cycle of 12:12 with lights onat 07:00 AM with free access to food and

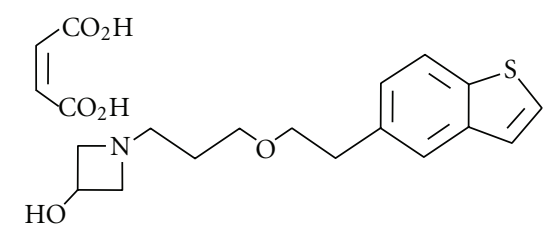

FIGURE 1: Chemical structure of T-817MA [1-\{3-[2-(1-benzothiophen-5-yl) ethoxy]propyl\} azetidin-3-ol maleate].

water. At the time of weaning (postnatal days; PD 21), the animals were grouped into four to six per treatment, in a cage with free access to food and water. The procedures complied with the National Institutes of Health Guide for the care and use of laboratory animals. All experiments were reviewed and approved by the Committee of Animal Research, University of Toyama.

2.2. Neonatal MK-801 Treatment. On PD 7, male pups (7$15 \mathrm{~g}$ ), born from 7 different female rats, were randomly divided into two groups; they received MK-801 (dizocilpine, $0.20 \mathrm{mg} / \mathrm{kg}$, s.c., Sigma-Aldrich, St. Louis, MO; MK-801 neonatal treatment group) or an equal volume of saline (control; vehicle group) once daily for 4 days. Pups received injections between 8:00-10:00.

2.3. Treatment with T-817MA, HPD, and RPD. The methods for administration of T-817MA were previously reported [25]. HPD and RPD were purchased from Dainippon Sumitomo Pharmaceuticals (Tokyo, Japan) and Janssen Pharmaceutical K. K. (Tokyo, Japan), respectively. On PD 49, animals were assigned to one of the following groups: saline-HPD group $(n=11)$, saline-RPD group $(n=10)$, MK801HPD group $(n=10)$, and MK801-RPD group $(n=11)$. HPD $(1.0 \mathrm{mg} / \mathrm{kg})$ and RPD $(1.0 \mathrm{mg} / \mathrm{kg})$ were administered s.c. once daily (8:00-10:00) for 14 days 7 (PD 49-62).

\subsection{Immunohistochemical Study}

2.4.1. Fixation. On PD 63 (24 hours after the last treatment), rats were euthanized by deep anesthesia with sodium pentobarbital (Nembutal, Abbott Laboratories, USA) before transcardial perfusion fixation with saline followed by $4 \%$ paraformaldehyde in $0.1 \mathrm{M}$ phosphate buffer. Brains were removed, postfixed in the same fixative at $4^{\circ} \mathrm{C}$, and were stored in $30 \%$ sucrose solution at $4{ }^{\circ} \mathrm{C}$ for cryoprotection. They were embedded in Tissue-Tek O.C.T. compound (Sakura Finetek USA, Torrance, CA, USA), rapidly frozen on dry ice, and were subsequently stored at $-80^{\circ} \mathrm{C}$.

2.4.2. Sectioning. Using a cryostat (Cryostats Leica CM 3050S, Leica Biosystems Nußloch GMBH, Nußloch, Germany), serial coronal sections of the brains were made with $30 \mu \mathrm{m}$ thickness through the mPFC and the hippocampus (AP from bregma $2.7 \mathrm{~mm}$ to $-3.6 \mathrm{~mm}$, resp.) [30]. The sections were mounted onto silage coated slides, dried for 4 hours at $38^{\circ} \mathrm{C}$, and immersed in $0.1 \mathrm{M}$ phosphate buffered saline containing $0.3 \%$ Triton $\mathrm{X}-100$ (PBS-T) at $4^{\circ} \mathrm{C}$. 


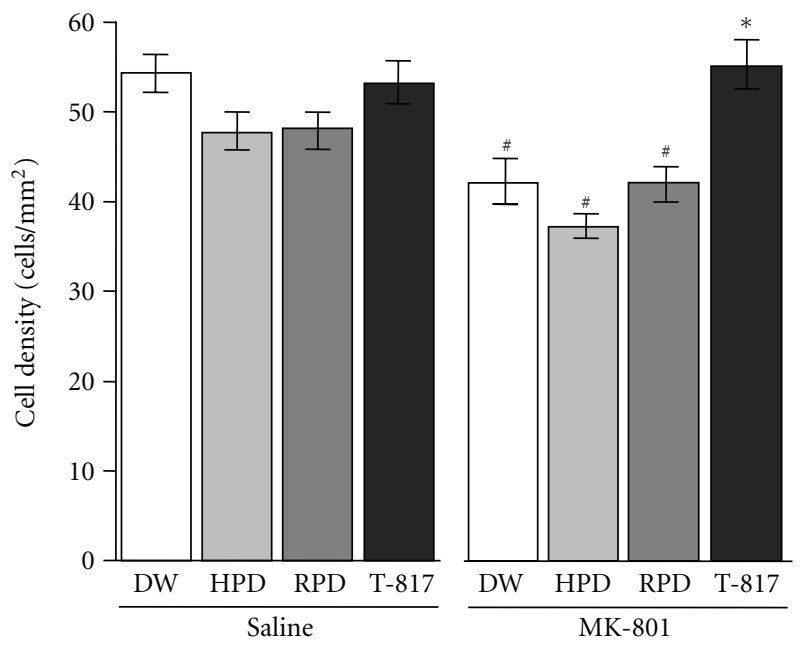

(a)
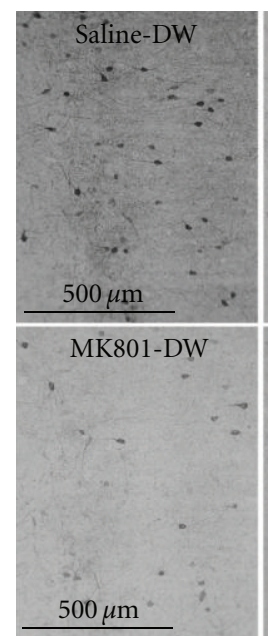

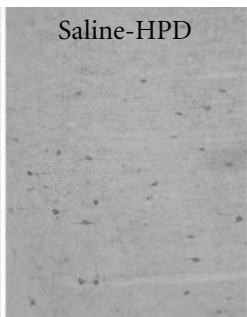

MK801-HPD
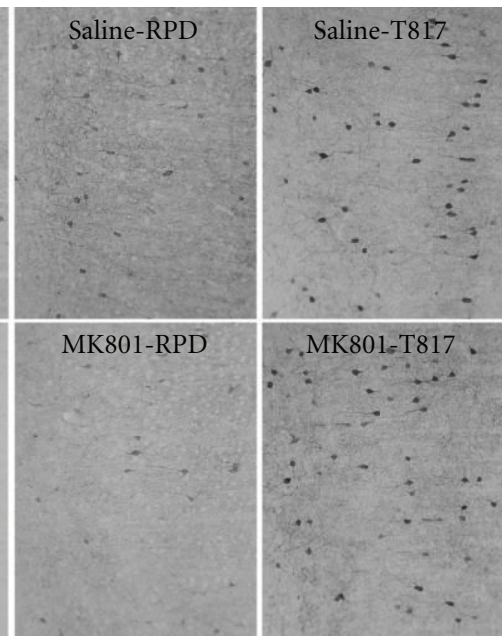

(b)

Figure 2: Effect of 14-day (PD 49-62) treatment with T-817MA, HPD or RPD on the density of PV-positive GABAergic interneurons (cells $\left./ \mathrm{mm}^{2}\right)$ in the mPFC. Data from DW group $(n=11)$, HPD group $(n=11)$, RPD group $(n=10)$, and T-817MA group $(n=11)$ treated neonatally with saline are shown in the left side. Data from DW group $(n=9)$, HPD group $(n=10)$, RPD group $(n=11)$, and T-817MA group $(n=9)$ treated neonatally with MK-801 are shown in the right side. Values are expressed as means \pm SEM. ${ }^{*} ; P<0.05$ as compared with saline-DW group. ${ }^{*} ; P<0.05$ as compared with MK-801-DW group. (b) Representative photomicrographs of PV-positive neurons in the mPFC from rats on PD 63.

2.4.3. Immunostaining. Sections were incubated for $10 \mathrm{~min}$ in a solution of $0.6 \%$ hydrogen peroxide in $10 \%$ methanol to eliminate endogenous peroxidases. After three consecutive 5 min washes with PBS-T, they were blocked for $60 \mathrm{~min}$ in PBS-T containing 2\% bovine serum albumin (Wako Pure Chemical Industries, Osaka, Japan). Then, the brain sections were incubated at $4^{\circ} \mathrm{C}$ overnight with a monoclonal antiparvalbumin antibody 8 (Sigma-Aldrich, St. Louis) $[1: 3000$ in antibody diluent (Dako, Glostrup, Denmark)]. After three consecutive $5 \mathrm{~min}$ washes with PBS-T, they were incubated for $60 \mathrm{~min}$ with horseradish peroxidase-labeled secondary antibody (Dako, Glostrup, Denmark). After washes with PBS-T, peroxidases were visualized using chromogen $3,3^{\prime}$ diaminobenzidine.
2.4.4. Quantification of Parvalbumin-Positive Cells. Quantification was performed in a blind fashion. All sections were observed using a light microscope $(\times 10$ objective, ECLIPSE E200, NIKON CORPORATION, Tokyo, Japan), and two sections which had intense signals of PV were analyzed for each brain area. PV-positive cell counts in the mPFC and hippocampus (whole part, as well as dentate gyrus, CA1, and CA2/CA3) were made bilaterally for two fields per section. The number of intensely stained neurons was counted within an $800 \times 800 \mu \mathrm{m}^{2}$ area in the $\mathrm{mPFC}$ and granule cell layers in the hippocampal subfields. Pictures for the counting were acquired with a light microscope equipped with a digital camera (Microscope Digital System Moticam2000, SHIMADZU RIKA CORPORATION, Tokyo, 
Japan) and a software (Motic Image Plus 2.1S, SHIMADZU RIKA CORPORATION, Tokyo, Japan). Data was expressed as the number of intensely stained neurons per $1 \mathrm{~mm}^{2}$.

2.5. Presentation of the Results and Statistics. Data were analyzed by analysis of variance (ANOVA) using SPSS software (version 19.0 J for Mac, IBM, Tokyo, Japan). Counts of PVpositive neurons were analyzed by one-way ANOVA followed by Bonferroni test.

\section{Results}

The results of T-817MA have been already reported [25]. One-way ANOVA revealed significant differences in the count of PV-positive neurons among 8 groups $(F=8.74, P<$ 0.001 ). Post hoc Bonferroni test indicated that neonatal MK801 treatment decreased PV-positive neurons (MK-801-DW group compared to saline-DW group, $P=0.007)$, which was ameliorated by T-817MA (MK-801-T817 group compared to MK-801-DW group, $P=0.006)$. HPD and RPD, on the other hand, did not influence the number of PV-positive neurons in MK-801-treated animals (MK-801-HPD group compared to MK-801-DW group, $P=1.00$; MK-801-RPD group compared to MK-801-DW group, $P=1.00$ ). Thus, HPD and RPD did not affect the number of PV-positive neurons in MK-801-treated animals in the MPFC, whereas T-817MA ameliorated its decrease (Figure 2(a)).

In the hippocampus, one-way ANOVA demonstrated significant differences in the count of PV-positive neurons among 8 groups $(F=13.54, P<0.001)$. Post hoc Bonferroni test showed that neonatal MK-801 treatment also decreased PV-positive neurons (MK801-DW group compared to saline-DW group, $P=0.016$ ), which was reversed by T-817MA (MK801-T817 group compared to MK801-DW group, $P=0.006)$. By contrast, HPD enhanced the MK801 -induced decrease in the number of PV-positive neurons (MK801-HPD group compared to MK801-DW group, $P=$ 0.042) (Figure 3(a)).

We subsequently analyzed the number of PV-positive neurons in the subregions of the hippocampus (DG, CA1, CA2/3 areas). In DG, one-way ANOVA revealed significant differences among 8 groups $(F=3.15, P=0.003)$ (Figures 4(a) and 4(d)). Post hoc test showed that PVpositive neurons were decreased in MK801-HPD group compared with saline-DW group $(P=0.005)$. In CA1, the number of PV-positive neurons was significantly different among the 8 groups $(F=3.11, P=0.006)$, due to a larger number of cells in MK801-T817 group compared to MK801-HPD group $(P=0.031)$. On the other hand, posthoc analysis revealed none of the MK-801 or antipsychotictreated groups was significantly different from saline-DW group or MK801-DW group (Figures 4(b) and 4(e)). There was a significant treatment effect in the CA2/3 area (oneway ANOVA; $F=12.05, P<0.001)$. Post hoc test demonstrated that neonatal MK-801 treatment decreased PV-positive neurons (MK801-DW group compared with saline-DW group, $P<0.001)$. T-817MA reversed the MK801-induced decline in the number of PV-positive neurons

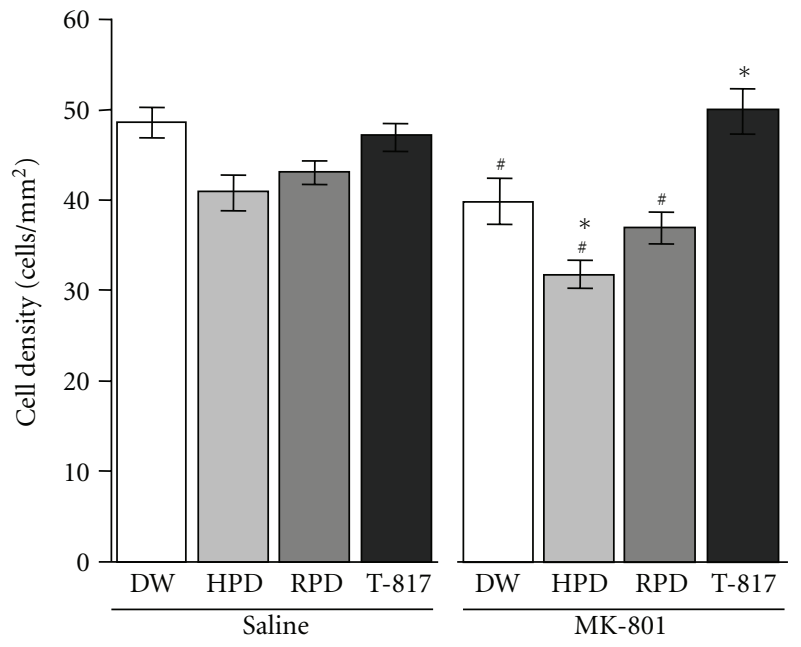

Figure 3: Effect of 14-day (PD 49-62) treatment with T-817MA, HPD, or RPD on the density of PV-positive GABAergic interneuron cells $\left(\right.$ cells $/ \mathrm{mm}^{2}$ ) in the hippocampus in rats that did or did not receive neonatal treatment with MK-801. DW group ( $n=12)$, HPD group $(n=10)$, RPD group $(n=10)$, and T-817MA group $(n=$ $11)$ treated neonatally with saline are shown in the left side. DW group ( $n=9)$, HPD group $(n=11)$, RPD group $(n=11)$, and T817MA group $(n=9)$ treated neonatally with MK-801 are shown in the right side. Data of saline group (open bars), HPD group (dark bars), RPD group (shaded bars), and T-817MA group (closed bars) are shown. Values are expressed as means \pm SEM. ${ }^{\#} P<0.05$ as compared with saline-DW group. ${ }^{*} ; P<0.05$ as compared with MK801-DW group.

(MK801-T817 group compared with MK801-DW group, $P=0.001)$. HPD and RPD, with or without combination with neonatal MK-801 treatment, decreased PV-positive neurons (in comparison with saline-DW group; $P<0.02$ ) (Figures 4(c) and 4(f)).

\section{Discussion}

The results of this study demonstrate the ability of T817MA to ameliorate the reduction of PV-positive GABA neurons in the brain of rats transiently exposed to NMDA receptor blockade at the neonatal period. On the other hand, both HPD and RPD did not show such effect in the mPFC of the neurodevelopmental model animals. In DG, PV-positive neurons were decreased by the combination of neonatal MK-801 administration and HPD treatment, compared with other treatment regimens. These model rats showed a decrease in the number of PV-positive neurons in the CA2/3, but not CA1 subfields, which was reversed by T817MA. HPD and RPD by themselves decreased PV-positive neurons in rats with or without neonatal exposure to MK801.

Treatment with HPD, RPD, or T-817MA was started around the period of puberty (PD 49 to 62 ) in our model animals. This timing was chosen based on the observations that 


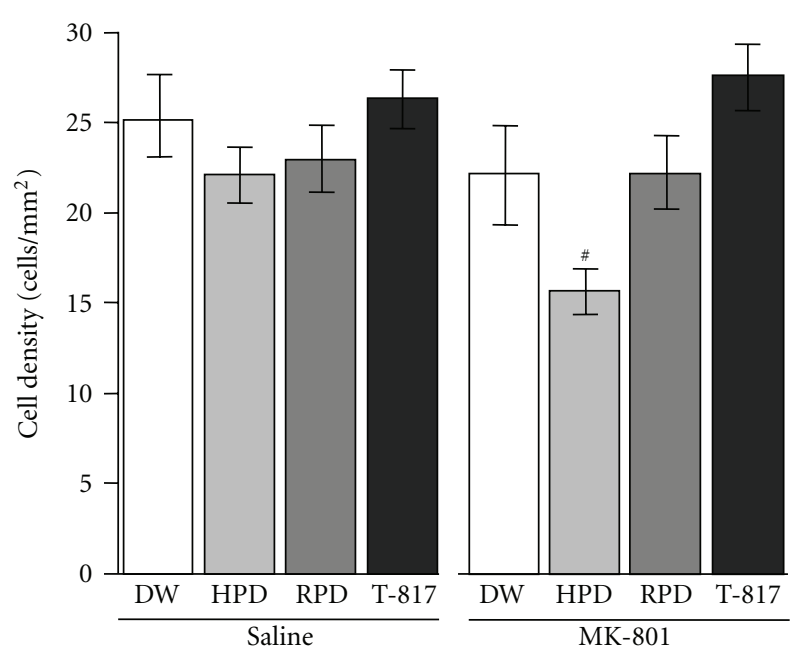

(a)

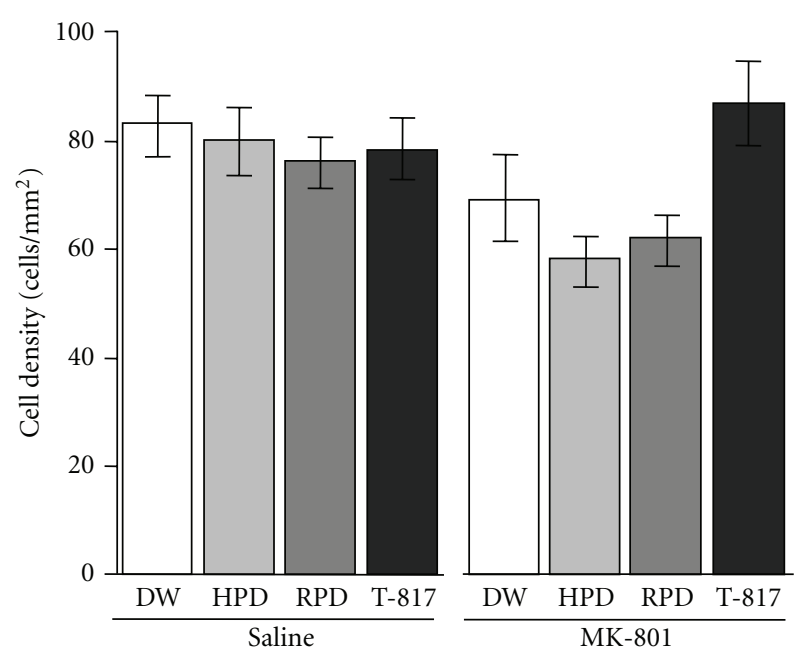

(b)

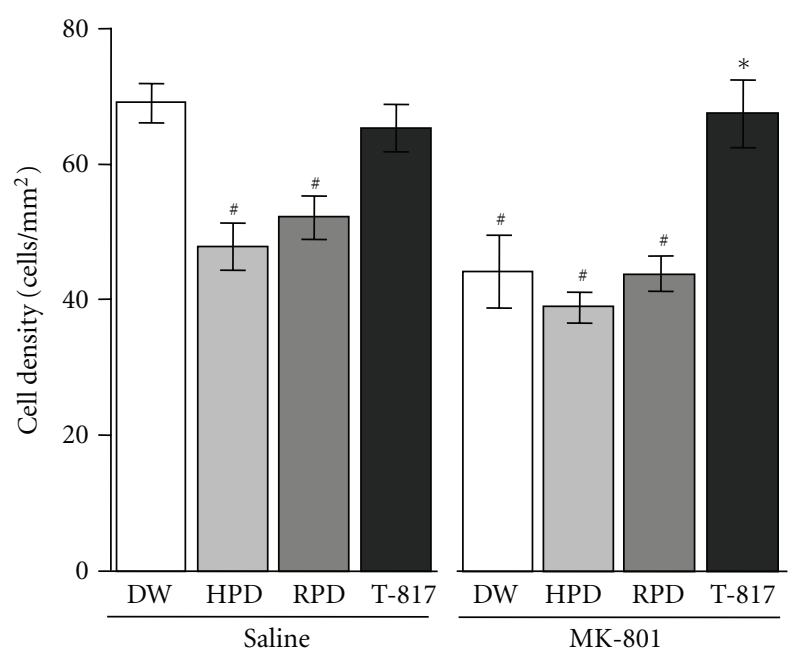

(c)

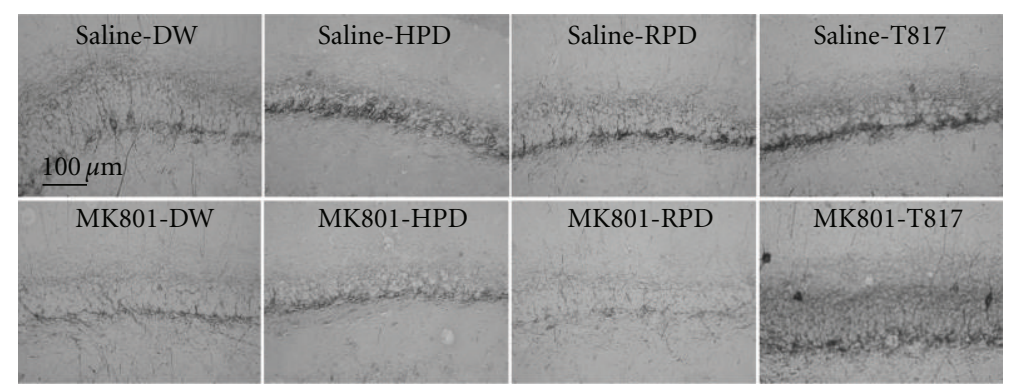

(d)

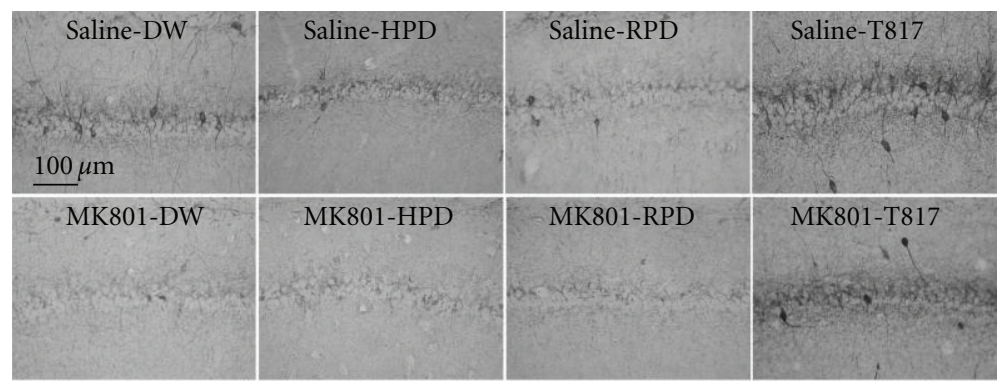

(e)

Figure 4: Continued. 


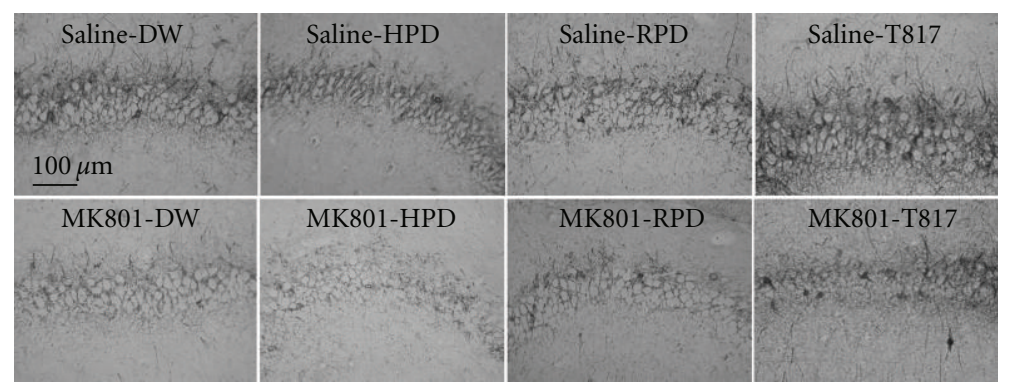

(f)

FIgURE 4: (a-c) Effect of 14-day (PD 49-62) treatment with T-817MA, HPD, or RPD on the density of PV-positive GABAergic interneuron cells $\left(\right.$ cells $/ \mathrm{mm}^{2}$ ) in the hippocampal subfields, that is, DG (a), CA1 (b), and CA2/3 (c), in rats that did or did not receive neonatal treatment with MK-801. DW group $(n=12)$, HPD group $(n=10)$, RPD group $(n=10)$, and T-817MA group $(n=11)$ treated neonatally with saline are shown in the left side. DW group $(n=9)$, HPD group $(n=11)$, RPD group $(n=11)$, and T-817MA group $(n=9)$ treated neonatally with MK-801 are shown in the right side. Data of saline group (open bars), HPD group (dark bars), RPD group (shaded bars) and T-817MA group (closed bars) are shown. Values are expressed as means \pm SEM. ${ }^{*} ; \quad<0.05$ as compared with saline-DW group. *; $P<0.05$ as compared with MK801-DW group. (d)-(f) Photomicrographs of PV-positive neurons in subregions of hippocampus from rats on PD 63; DG (d), CA1 (e), and CA2/3 (f).

(1) patients with schizophrenia manifest psychosis around puberty $[1,2]$, and (2) our model rats elicit disruption of sensorimotor gating and increased methamphetamineinduced locomotor activity at postpuberty (PD 63), but not prepuberty (PD 35) $[28,29]$.

4.1. Effect of T-817MA. T-817MA elicits neuroprotective effects against amyloid- $\beta$ or $\mathrm{H}_{2} \mathrm{O}_{2}$-induced neurotoxicity, while decrease of glutathione (GSH) levels induced by $\mathrm{H}_{2} \mathrm{O}_{2}$ exposure was suppressed by pretreatment with T-817MA [23]. GSH is an important intracellular antioxidant that protects the neurons against a variety of reactive oxygen species (ROS) [31]. In addition, this agent reduced attenuation of ROS production in mitochondria [32]. T-817MA has been also shown to facilitate neurogenesis in vitro. Thus, it promotes neurite outgrowth and increases the amount of growth-associated protein 43 in hippocampal slice cultures and neuronal reaggregation culture [23]. T-817MA has been also demonstrated to increase PSA-NCAM, a marker of cell proliferation, and bromodeoxyuridine (BrdU)-positive cells in the dentate gyrus (DG) of rats that received continuous $\mathrm{A} \beta$ infusion into the cerebral ventricles [22]. These findings indicate that T-817MA stimulates proliferation of neural progenitor cells and enhances survival of the newly generated cells in the DG against neurotoxicity [22].

Behaviorally, T-817MA has been shown to ameliorate memory impairment in rats receiving intracerebroventricular infusion of $\mathrm{A} \beta[21,22]$. Thus, T-817MA has been demonstrated to improve cognitive impairment, as measured by the Y-maze task, in mice expressing FTDP17 human P301L mutant tau [24]. This agent also corrects tau-induced synaptic abnormalities and enhances synaptic terminal density in the hippocampus [24].

The protective effect of T-817MA against human tau (h-tau42)-mediated axonal/synaptic dysfunction has been demonstrated by means of tau-mediated synaptic block, synaptic vesicle aggregation, and decreased h-tau 42 phosphorylation [33]. Moreover, T-817MA has been suggested to protect against Parkinson's disease. For example, it can prevent 1-methyl-4-phenyl-1,2,3,6-tetrahydropyridine (MPTP)-induced dopaminergic neurotoxicity in C57BL/6J mice [34]. MPTP impairs mitochondrial respiration by inhibiting complex 1 and causes dopaminergic neurotoxicity leading to behavioral impairment similar to the features of Parkinson's disease $[35,36]$. Pretreatment with T-817MA attenuates MPTP-induced decrease in dopamine levels and tyrosine hydroxylase immunostaining in the substantia nigra (SNc) and striatum [34]. It also ameliorates impairment of rotarod performance, a measure of coordinated motor skills [34]. Taken together, T-817MA is thought to exert neuroprotective effects against neurotoxicity caused by antioxidative insults.

4.2. Effects of HPD and RPD. Some researchers report the effects of HPD or RPD on decreased PV-positive neurons induced by NMDA antagonists $[37,38]$. Thus, coadministration of HPD attenuated the detrimental effect of MK-801 on $\mathrm{PV}$-positive neurons in the hippocampus of juvenile rats, but markedly reduced immunoreactivity to PV in the prefrontal cortex [37]. Concurrent administration of RPD with PCP did not protect against reduction in the expression of PVpositive neurons in the prefrontal cortex [38]. These previous findings are partly consistent with the results of our data (Figure 2(a)). Moreover, it is reported that pretreatment with olanzapine prevented the apoptosis in the frontal cortex of rat induced by $10 \mathrm{mg} / \mathrm{kg}$ PCP administration on PD 7, 9, and 11 [39]. This finding suggests that perinatal administration of some SGAs may block apoptosis.

In sum, some of the existing antipsychotic drugs, such as HPD and RPD, show the limited ability to protect PVpositive interneurons in the $\mathrm{mPFC}$ and hippocampus in animals that received transient excitotoxic insults in the neonatal period. 


\section{Conclusion}

Using a neurodevelopmental animal model, we have demonstrated the ability of T-817MA to ameliorate histological abnormalities in brain areas responsible for cognitive disturbances of schizophrenia, while some widely used antipsychotics did not show such effect. To our knowledge, T-817MA is the first agent to reverse the reduction in the number of PV-positive GABA interneurons induced by blockade of NMDA receptors. Our findings may provide a novel approach for the treatment of the core features of schizophrenia, such as cognitive deficits.

\section{Acknowledgments}

This work was supported by Toyama Chemical Co., Ltd., Grant-in-Aid from Japan Society for the Promotions of Sciences, and Health and Labour Sciences Research Grants. The authors gratefully acknowledge the insightful comments and discussions by Dr. Noboru Iwakami. Special thanks are given to Mr. Hiroshi Hattori for his technical support. The authors would also be grateful to Professor Michio Suzuki for his support.

\section{References}

[1] E. J. Bromet and S. Fennig, "Epidemiology and natural history of schizophrenia," Biological Psychiatry, vol. 46, no. 7, pp. 871$881,1999$.

[2] D. A. Lewis and J. A. Lieberman, "Catching up on schizophrenia: natural history and neurobiology," Neuron, vol. 28, no. 2, pp. 325-334, 2000.

[3] J. A. Lieberman, D. Perkins, A. Belger et al., "The early stages of schizophrenia: speculations on pathogenesis, pathophysiology, and therapeutic approaches," Biological Psychiatry, vol. 50, no. 11, pp. 884-897, 2001.

[4] R. Freedman, "Schizophrenia," The New England Journal of Medicine, vol. 349, no. 18, pp. 1738-1749, 2003.

[5] D. R. Weinberger, "Implications of normal brain development for the pathogenesis of schizophrenia," Archives of General Psychiatry, vol. 44, no. 7, pp. 660-669, 1987.

[6] D. R. Weinberger, "Neurodevelopmental perspectives on schizophrenia," in Psychopharmacology, the Forth Generation of Progress, F. E. Bloom and D. J. Kupfer, Eds., pp. 1171-1183, Raven Press, York, NY, USA, 1995.

[7] J. A. Lieberman, L. F. Jarskog, and D. Malaspina, "Preventing clinical deterioration in the course of schizophrenia: the potential for neuroprotection," Journal of Clinical Psychiatry, vol. 67, no. 6, pp. 983-990, 2006.

[8] J. A. Lieberman, "Is schizophrenia a neurodegenerative disorder? A clinical and neurobiological perspective," Biological Psychiatry, vol. 46, no. 6, pp. 729-739, 1999.

[9] L. F. Jarskog, L. A. Glantz, J. H. Gilmore, and J. A. Lieberman, "Apoptotic mechanisms in the pathophysiology of schizophrenia," Progress in Neuro-Psychopharmacology and Biological Psychiatry, vol. 29, no. 5, pp. 846-858, 2005.

[10] M. P. Mattson and W. Duan, “'Apoptotic' biochemical cascades in synaptic compartments: roles in adaptive plasticity and neurodegenerative disorders," Journal of Neuroscience Research, vol. 58, no. 1, pp. 152-166, 1999.
[11] J. L. Rapoport, A. M. Addington, S. Frangou, and M. R. C. Psych, "The neurodevelopmental model of schizophrenia: update 2005," Molecular Psychiatry, vol. 10, no. 5, pp. 434-449, 2005.

[12] S. H. Fatemi and T. D. Folsom, "The neurodevelopmental hypothesis of Schizophrenia, revisited," Schizophrenia Bulletin, vol. 35, no. 3, pp. 528-548, 2009.

[13] A. M. Mcintosh, D. C. Owens, W. J. Moorhead et al., "Longitudinal volume reductions in people at high genetic risk of schizophrenia as they develop psychosis," Biological Psychiatry, vol. 69, no. 10, pp. 953-958, 2011.

[14] J. A. Lieberman, F. P. Bymaster, H. Y. Meltzer et al., "Antipsychotic drugs: comparison in animal models of efficacy, neurotransmitter regulation, and neuroprotection," Pharmacological Reviews, vol. 60, no. 3, pp. 358-403, 2008.

[15] D. A. Lewis, T. Hashimoto, and D. W. Volk, "Cortical inhibitory neurons and schizophrenia," Nature Reviews Neuroscience, vol. 6, no. 4, pp. 312-324, 2005.

[16] F. M. Benes and S. Berretta, "GABAergic interneurons: implications for understanding schizophrenia and bipolar disorder," Neuropsychopharmacology, vol. 25, no. 1, pp. 1-27, 2001.

[17] C. L. Beasley and G. P. Reynolds, "Parvalbumin-immunoreactive neurons are reduced in the prefrontal cortex of schizophrenics," Schizophrenia Research, vol. 24, no. 3, pp. 349-355, 1997.

[18] C. L. Beasley, Z. J. Zhang, I. Patten, and G. P. Reynolds, "Selective deficits in prefrontal cortical GABAergic neurons in schizophrenia defined by the presence of calcium-binding proteins," Biological Psychiatry, vol. 52, no. 7, pp. 708-715, 2002.

[19] E. F. Torrey, B. M. Barci, M. J. Webster, J. J. Bartko, J. H. Meador-Woodruff, and M. B. Knable, "Neurochemical markers for schizophrenia, bipolar disorder, and major depression in postmortem brains," Biological Psychiatry, vol. 57, no. 3, pp. 252-260, 2005.

[20] Z. J. Zhang and G. P. Reynolds, "A selective decrease in the relative density of parvalbumin-immunoreactive neurons in the hippocampus in schizophrenia," Schizophrenia Research, vol. 55, no. 1-2, pp. 1-10, 2002.

[21] P. T. H. Nguyen, T. Kimura, A. H. Son, H. T. Anh, T. Ono, and H. Nishijo, "Ameliorative effects of a neuroprotective agent, T-817MA, on place learning deficits induced by continuous infusion of amyloid- $\beta$ peptide (1-40) in rats," Hippocampus, vol. 17, no. 6, pp. 443-455, 2007.

[22] T. Kimura, P. T. H. Nguyen, S. A. Ho, A. H. Tran, T. Ono, and H. Nishijo, "T-817MA, a neurotrophic agent, ameliorates the deficits in adult neurogenesis and spatial memory in rats infused i.c.v. with amyloid-ß peptide," British Journal of Pharmacology, vol. 157, no. 3, pp. 451-463, 2009.

[23] K. Hirata, H. Yamaguchi, Y. Takamura et al., "A novel neurotrophic agent, T-817MA [1-3-[2-(1-benzothiophen-5-yl) ethoxy] propyl-3-azetidinol maleate], attenuates amyloid- $\beta$ induced neurotoxicity and promotes neurite outgrowth in rat cultured central nervous system neurons," Journal of Pharmacology and Experimental Therapeutics, vol. 314, no. 1, pp. 252-259, 2005.

[24] T. Fukushima, A. Nakamura, N. Iwakami et al., "T-817MA, a neuroprotective agent, attenuates the motor and cognitive impairments associated with neuronal degeneration in P301L tau transgenic mice," Biochemical and Biophysical Research Communications, vol. 407, no. 4, pp. 730-734, 2011.

[25] T. Uehara, T. Sumiyoshi, H. Hattori et al., "T-817MA, a novel neurotrophic agent, ameliorates loss of GABAergic parvalbumin-positive neurons and sensorimotor gating deficits in rats transiently exposed to MK-801 in the neonatal 
period," Journal of Psychiatric Research, vol. 46, no. 5, pp. 622629, 2012.

[26] T. Seo, T. Sumiyoshi, M. Tsunoda et al., "T-817MA, a novel neurotrophic compound, ameliorates phencyclidine-induced disruption of sensorimotor gating," Psychopharmacology, vol. 197, no. 3, pp. 457-464, 2008.

[27] T. Uehara, H. Itoh, T. Matsuoka et al., "Effect of transient blockade of $\mathrm{N}$-methyl-D-aspartate receptors at neonatal stage on stress-induced lactate metabolism in the medial prefrontal cortex of adult rats: role of 5-HT1A receptor agonism," Synapse, vol. 66, no. 5, pp. 408-417, 2012.

[28] T. Uehara, T. Sumiyoshi, T. Seo et al., "Long-term effects of neonatal MK-801 treatment on prepulse inhibition in young adult rats," Psychopharmacology, vol. 206, no. 4, pp. 623-630, 2009.

[29] T. Uehara, T. Sumiyoshi, T. Seo et al., "Neonatal exposure to MK-801, an N-methyl-d-aspartate receptor antagonist, enhances methamphetamine-induced locomotion and disrupts sensorimotor gating in pre- and postpubertal rats," Brain Research, vol. 1352, pp. 223-230, 2010.

[30] G. Paxinos and C. Watson, The Rat Brain in Stereotaxic Coordinates, Academic Press, Sydney, 1998.

[31] J. B. Schulz, J. Lindenau, J. Seyfried, and J. Dichgans, "Glutathione, oxidative stress and neurodegeneration," European Journal of Biochemistry, vol. 267, no. 16, pp. 4904-4911, 2000.

[32] T. Fukushima, M. Koide, Y. Ago, A. Baba, and T. Matsuda, "T-817MA, a novel neurotrophic agent, improves sodium nitroprusside-induced mitochondrial dysfunction in cortical neurons," Neurochemistry International, vol. 48, no. 2, pp. 124$130,2006$.

[33] H. Moreno, S. Choi, E. Yu et al., "Blocking effects of human Tau on squid giant synapse transmission and its prevention by T-817 MA," Frontiers in Synaptic Neuroscience, vol. 3, no. 3, 2011.

[34] T. Kawasaki, Y. Ago, T. Kitao et al., "A neuroprotective agent, T-817MA (1-3-[2-(1-benzothiophen-5-yl)ethoxy]propyl azetidin-3-ol maleate), prevents 1-methyl-4-phenyl-1,2,3,6-tetrahydropyridineinduced neurotoxicity in mice," Neuropharmacology, vol. 55, no. 5, pp. 654-660, 2008.

[35] A. H. V. Schapira, J. M. Cooper, D. Dexter, J. B. Clark, P. Jenner, and C. D. Marsden, "Mitochondrial Complex I deficiency in Parkinson's disease," Journal of Neurochemistry, vol. 54, no. 3, pp. 823-827, 1990.

[36] M. Sedelis, R. K. W. Schwarting, and J. P. Huston, "Behavioral phenotyping of the MPTP mouse model of Parkinson's disease," Behavioural Brain Research, vol. 125, no. 1-2, pp. 109$125,2001$.

[37] I. Braun, J. Genius, H. Grunze, A. Bender, H. J. Möller, and D. Rujescu, "Alterations of hippocampal and prefrontal GABAergic interneurons in an animal model of psychosis induced by NMDA receptor antagonism," Schizophrenia Research, vol. 97, no. $1-3$, pp. 254-263, 2007.

[38] C. E. McKibben, T. A. Jenkins, H. N. Adams, M. K. Harte, and G. P. Reynolds, "Effect of pretreatment with risperidone on phencyclidine-induced disruptions in object recognition memory and prefrontal cortex parvalbumin immunoreactivity in the rat," Behavioural Brain Research, vol. 208, no. 1, pp. 132-136, 2010.

[39] C. Wang, J. McInnis, M. Ross-Sanchez, P. Shinnick-Gallagher, J. L. Wiley, and K. M. Johnson, "Long-term behavioral and neurodegenerative effects of perinatal phencyclidine administration: implications for schizophrenia," Neuroscience, vol. 107, no. 4, pp. 535-550, 2001. 


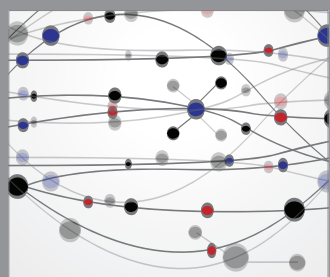

The Scientific World Journal
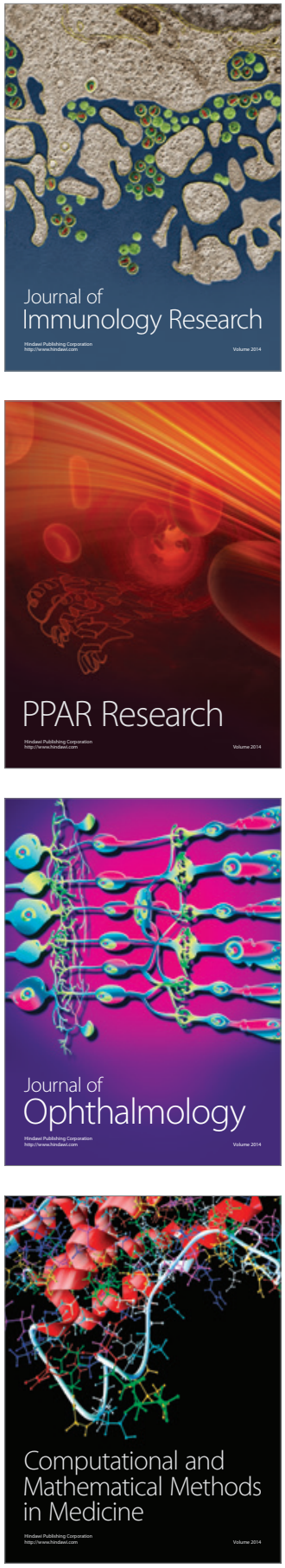

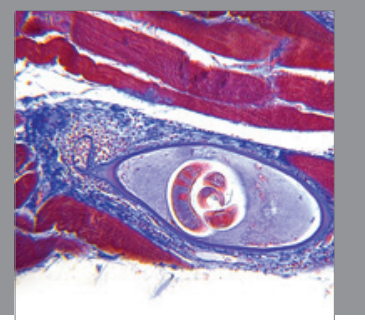

Gastroenterology

Research and Practice
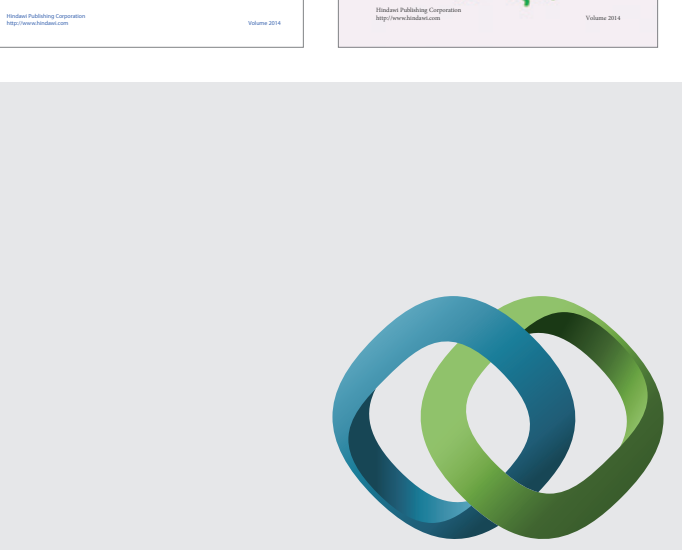

\section{Hindawi}

Submit your manuscripts at

http://www.hindawi.com
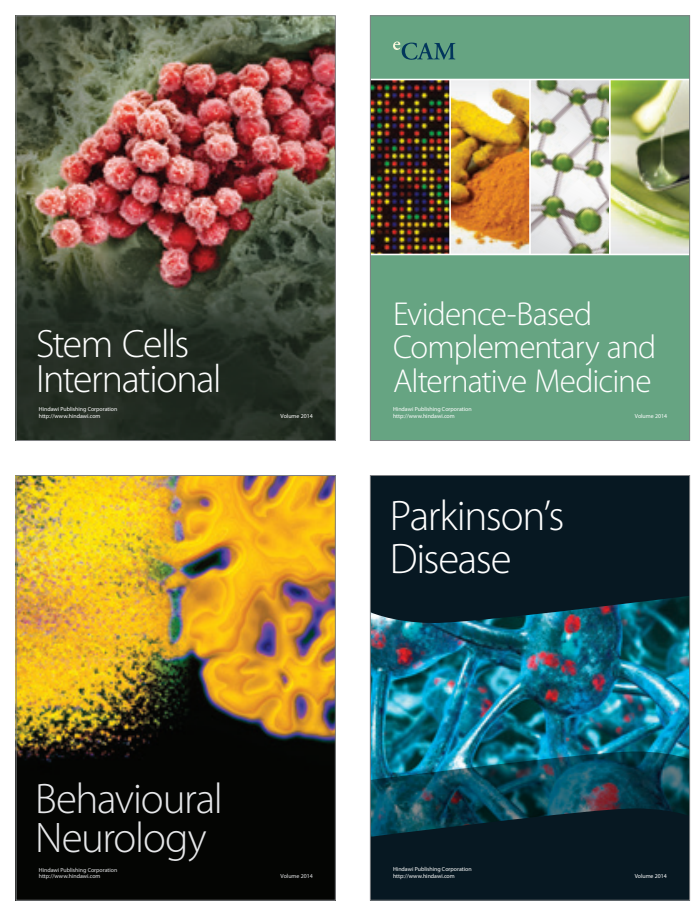

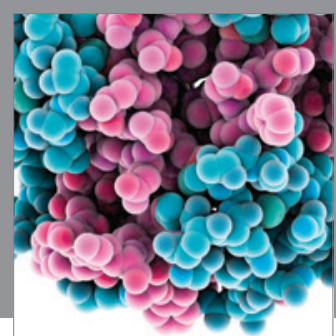

Journal of
Diabetes Research

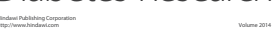

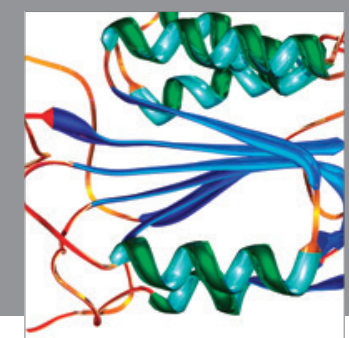

Disease Markers
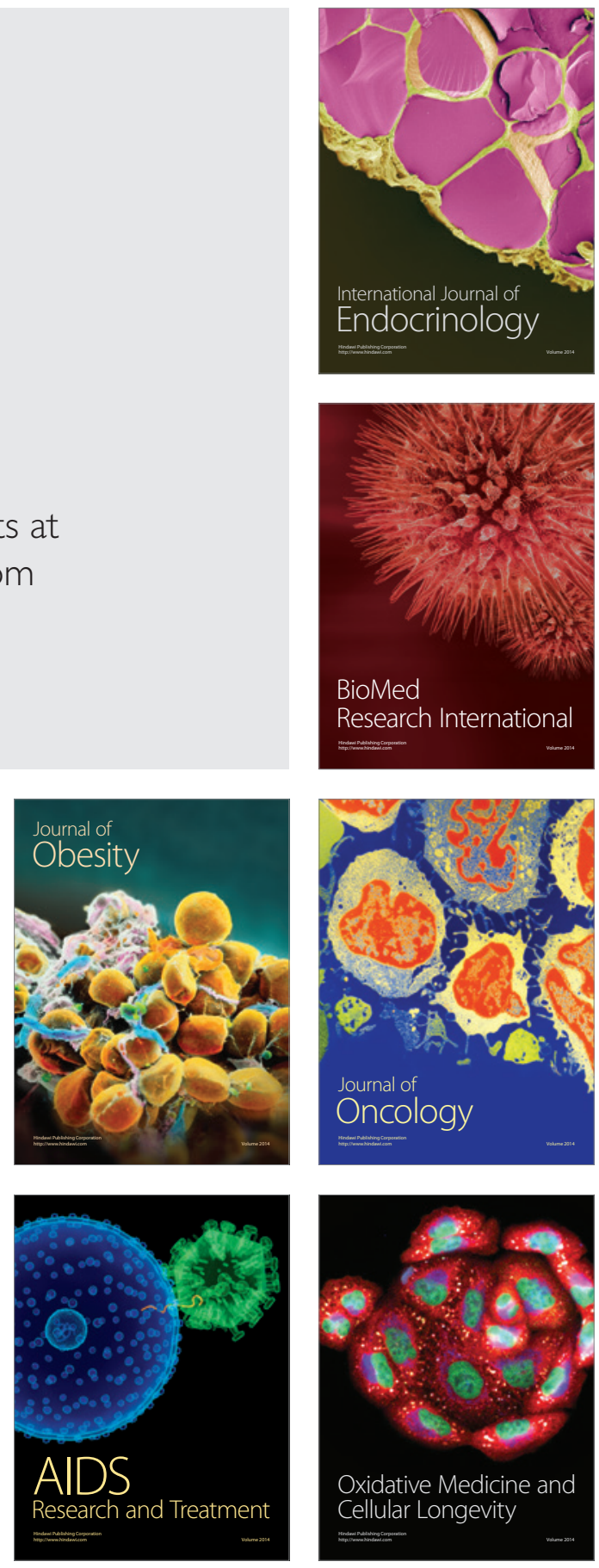